not distress themselves, for the approach of the new book is still essentially one of comparison. After two excellently-written general chapters on biosynthesis and on function, the author moves on to consider, separately, all aspects of the carotenoids of the photosynthetic tissues of seed-bearing plants, and of those of their reproductive tissues and roots. Then he deals, again in separate chapters, with the carotenoids formed by mosses, liverworts and sporebearing vascular plants, by algae, by fungi, by the non-photosynthetic and by the photosynthetic bacteria. For each group of organisms, a consideration of distribution is followed by a description of those features of carotenoid function and biosynthesis which are peculiar to that group, thus supplementing the earlier description of common features. The control of carotenogenesis by genetic, environmental and chemical means is discussed whenever appropriate.

Many carotenoid biochemists (and others) have tried to use correlations of (a) carotenoid structure and distribution with (b) plant classification as a retrospective comment on how plant evolution may have taken place. Goodwin's final chapter, "Biogeochemistry", describes the few results but considerable potential of carotenoid analysis as a means of demonstrating how plant evolution actually occurred.

This really is an excellent book; it is not only scholarly and written with great clarity, but also well planned. The basic structures of some 194 carotenoids are included, among other line illustrations, in addition to a number of apposite electron micrographs. But perhaps one of the most important features of the book is the number and quality of the comparative tables -86 of them. Of these, the most impressive covers seven pages and shows the distribution of 67 fruit carotenoids throughout the 160 higher plant species examined to date. Fully referenced as it is, it could stand on its own as a review article. Also packed into the book's 377 pages are a general index and a particularly useful species index.

Although several excellent books on carotenoids and other plant pigments have appeared over the past decade, Goodwin's new volume is clearly set to be the most authoritative, comprehensive and comprehensible work available on their biochemistry. There are few really readable reference works on any discipline, so those of us with an interest in carotenoids are particularly fortunate. I am sure this book will stimulate even more interest in carotenoid biochemistry, for there is much in it to appeal to the intellect and imagination of undergraduates, research workers and practitioners in all areas of biological science.

B.H. Davies is Senior Lecturer in Biochemistry at The University College of Wales, Aberystwyth.

\title{
How do electrons get from here to there?
}

\section{Thomas J. Meyer}

Electron Transfer Reactions. By R.D. Cannon. Pp.351. ISBN 0-408-10646-8. (Butterworth: 1981.) £32, \$84.95.

COMPARED to most chemical reactions, electron transfer is a simple process. An electron hops from one chemical site to another, in many cases without significant change in structure. Even so, electron transfer is at the heart of many critical issues in chemistry, physics and biology, and its study is currently enjoying a remarkable vitality. As evidence note the book Tunnelling in Biological Systems edited by B. Chance et al. (Academic Press, 1979) which provides an account of a meeting on the same theme held four years ago in Philadelphia.

The origins of this new interest in electron transfer are twofold. The first is the existence of a useful interplay between theory and experiment which is leading to a "solution" of sorts - a solution in the sense that the microscopic factors controlling rates of electron transfer reactions can be understood by chemists, biochemists and physicists based on wellestablished ideas in chemistry and physics. The second is the spill-over effect that these advances will and are having on related problems in chemistry, physics, biology and photochemistry.

Having said all of this, it must be added that the subject can be a source of extreme frustration. For a chemist, much of the theory - which has its origins in work on defects in solids, radiationless processes or nonequilibrium thermodynamics - is barely comprehensible. Also, there are far too many experimental results and yet too few critical experiments, the result being that attempts to generalize often lead to confusion.

Into this setting comes Electron Transfer Reactions by R.D. Cannon. The first thing to be said about the book is that it is a real work of scholarship. A diverse and voluminous literature is brought together in a single volume. The second is that it is a book by a chemist written for other chemists. There are introductory chapters on electron transfer in the gas phase and in the solid state, but the emphasis is on electron transfer in solution, mostly in aqueous solution and predominantly involving transition metal ions.

Cannon's synthesis of the available experimental data is a creative act, and much thought has been given to both their organization and interpretation. The result is generally good - for example the introduction of specimen calculations, the systematization of such diverse topics as

Richard Mabey's The Common Ground (for review see Nature 286,87 ) has recently appeared in paperback. The book is published by Arrow and costs £1.95. free energy profiles, multiple electron steps and successor complexes - but occasionally bad - the use of symbols instead of real examples to illustrate reaction types is sometimes hard to follow, and the development of the theoretical background is somewhat inconsistent and confusing. The book includes chapters on reaction pathways and mechanisms, theory, electron transfer through ligand bridges and optical electron transfer, in addition to the chapters on electron transfer in the gas phase and the solid state.

One limitation is that no attempt is made to develop the emerging generalities alluded to above. For example, there is little mention of either photochemistry or of electron transfer in biological systems. It is also true that the author is occasionally guilty of focusing on the bizarre or the unusual at the expense of the more general. However, I find that Cannon's book serves admirably in its role as a sophisticated guide to electron transfer in solution. It is a valuable and badly needed addition to the literature, since its predecessor, Mechanisms of Electron Transfer by Reynolds and Lumry (The Ronald Press, 1966), is now well out of date.

Thomas J. Meyer is Professor of Chemistry at the University of North Carolina, Chapel Hill.
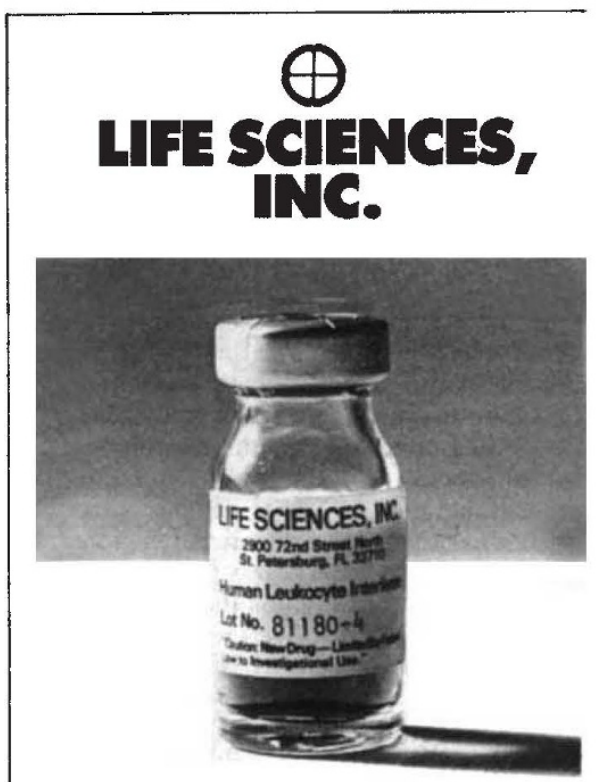

\section{Supplying Human Leukocyte Interferon for clinical tests and research.}

2900:72nd Street North St. Petersburg, Florida 33710 (813) 345-9371

Circle No.06 on Reader Enquiry Card. 